\title{
FINAL REPORT FOR DOE GRANT DE-FG02-03ER25579: "DEVELOPMENT OF HIGH-ORDER ACCURATE INTERFACE TRACKING ALGORITHMS AND IMPROVED CONSTITUTIVE MODELS FOR PROBLEMS IN CONTINUUM MECHANICS WITH APPLICATIONS TO JETTING"
}

\author{
PROFESSOR ELBRIDGE GERRY PUCKETT \\ DEPARTMENT OF MATHEMATICS \\ UNIVERSITY OF CALIFORNIA \\ DAVIS, CA, 95616 \\ PROFESSOR GREGORY HALE MILLER \\ DEPARTMENT OF CHEMICAL ENGINEERING \\ UNIVERSITY OF CALIFORNIA \\ DAVIS, CA, 95616
}

\section{Introduction}

Much of the work conducted under the auspices of DE-FG02-03ER25579 was characterized by an exceptionally close collaboration with researchers at the Lawrence Berkeley National Laboratory (LBNL). For example, Andy Nonaka, one of Professor Miller's graduate students in the Department of Applied Science at U. C. Davis (UCD) wrote his PhD thesis [22] in an area of interest to researchers in the Applied Numerical Algorithms Group (ANAG), which is a part of the National Energy Research Supercomputer Center (NERSC) at LBNL. Dr. Nonaka collaborated closely with these researchers and subsequently published the results of this collaboration jointly with them, one article in a peer reviewed journal article [23] and one paper in the proceedings of a conference [32]. Dr. Nonaka is now a research scientist in the Center for Computational Sciences and Engineering (CCSE), which is also part of the National Energy Research Supercomputer Center (NERSC) at LBNL.

This collaboration with researchers at LBNL also included having one of Professor Puckett's graduate students in the Graduate Group in Applied Mathematics (GGAM) at UCD, Sarah Williams, spend the summer working with Dr. Ann Almgren, who is a staff scientist in CCSE. As a result of this visit Sarah decided work on a problem suggested by the head of CCSE, Dr. John Bell, for her $\mathrm{PhD}$ thesis. Having finished all of the coursework and examinations required for a $\mathrm{PhD}$, Sarah stayed at LBNL to work on her thesis under the guidance of Dr. Bell. Sarah finished her PhD thesis in June of 2007 [39]. Writing a $\mathrm{PhD}$ thesis while working at one of the University of California (UC) managed DOE laboratories is long established tradition at UC and Professor Puckett has always encouraged his students to consider doing this.

Another one of Professor Puckett's graduate students in the GGAM at UCD, Christopher Algieri, was partially supported with funds from DE-FG02-03ER25579 while he wrote his MS thesis [1] in which he analyzed and extended work originally published by Dr. Phillip Colella, the head of ANAG, and some of his colleagues. Chris Algieri is now employed as a staff member in Dr. Bill Collins' Climate Science Department in the Earth Sciences Division at LBNL working with computational models of climate change. Chris is planning to write a $\mathrm{PhD}$ thesis while employed as a staff scientist in Dr. Collins' group. The plan is for his thesis topic to be in an area that is of interest to both Dr. Collins and Dr. Colella. 
Finally, it should be noted that the work conducted by Professor Puckett and his students Sarah Williams and Chris Algieri and described in this final report for DOE grant \# DE-FG02-03ER25579 is closely related to work performed by Professor Puckett and his students under the auspices of Professor Puckett's DOE SciDAC grant \# DE-FC02-01ER25473 "An Algorithmic and Software Framework for Applied Partial Differential Equations: A DOE SciDAC Integrated Software Infrastructure Center (ISIC)". Dr. Colella was the lead PI for this SciDAC grant, which was comprised of several research groups from DOE national laboratories and five university PI's from five different universities. In theory Professor Puckett tried to use funds from the SciDAC grant to support work directly involved in implementing algorithms developed by members of his research group at UCD as software that might be of use to Puckett's SciDAC CoPIs. (For example, see the work reported in Section 2.2.2 below.) However, since there is considerable lead time spent developing such algorithms before they are ready to become 'software' and research plans and goals change as the research progresses, Professor Puckett supported each member of his research group partially with funds from the SciDAC APDEC ISIC DE-FC02-01ER25473 and partially with funds from this DOE MICS grant DE-FG02-03ER25579. This has necessarily resulted in a significant overlap of project areas that were funded by both grants.

In particular, Both Sarah Williams and Chris Algieri were supported partially with funds from grant \# DE-FG02-03ER25579, for which this is the final report, and in part with funds from Professor Puckett's DOE SciDAC grant \# DE-FC02-01ER25473. For example Sarah Williams received support from DE-FC02-01ER25473 and DE-FG02-03ER25579, both while at UCD taking classes and writing her MS thesis [38] and during the first year she was living in Berkeley and working at LBNL on her PhD thesis. In Chris Algieri's case he was at UCD during the entire time he received support from both grants. More specific details of their work are included in the report below.

\section{Work Performed by Professor Puckett and Members of His Research Group}

\subsection{Development and Analysis of Second-Order Accurate Volume-of-Fluid Methods.}

\subsubsection{A New Class of Second-order Accurate Volume-of-Fluid Methods Interface Tracking Meth-} ods

During the funding period covered by DE-FG02-03ER25579 Professor Puckett revised and published a paper [26] in which he and his former MS and PhD student Dr. James E. Pilliod introduced a new class of second-order accurate volume-of-fluid (VOF) interface reconstruction algorithms that have come to be known generically as Piecewise Linear Interface Calculation (PLIC) methods. Although there had been previously published versions of piecewise linear VOF interface reconstruction algorithms, in [26] Pilliod and Puckett showed that these previously published algorithms were all first-order accurate. In [26] Pilliod and Puckett introduced two piecewise linear VOF interface reconstruction algorithms, the "Least Squares VOF Interface Reconstruction Algorithm" (LVIRA) and the "Efficient Least Squares VOF Interface Reconstruction Algorithm" (ELVIRA) and demonstrated on various test problems that both were second-order accurate. ${ }^{1}$

In general, a numerical method for modeling the motion in time of the interface between two materials consists of two separate algorithms. First, one must have a numerical representation of the interface at each fixed moment in time. A method for doing this is called an interface reconstruction algorithm. Then, since the interface is moving in time, one must also have an algorithm for tracking or advecting the motion of the interface in time. In [26] Pilliod and Puckett also introduced a new

\footnotetext{
${ }^{1}$ All of Pilliod's work reported on in [26], which was an outgrowth of his Masters thesis [24] in the GGAM at UCD, predated the period covered by DE-FG02-03ER25579. After finishing his PhD thesis [25] Pilliod spent several years as a postdoc Applied Numerical Algorithms Group at LBNL working with Dr. Colella and his ANAG colleagues and then took a job in the 'dot.com' industry. However, Professor Puckett was partially supported by DE-FG02-03ER25579 while he was revising [26] for publication.
} 
second-order accurate volume-of-fluid advection algorithm that was based on ideas developed by John Bell and his coauthors in [5].

Volume-of-fluid interface tracking algorithms are important for a variety of reasons. From DOE's (and the nation's) point of view, perhaps the most important reason is that most, if not all, of the 'production' codes used by scientists at the Los Alamos, Lawrence Livermore, and other DOE National Laboratories to model the movement of a large number of distinct materials are based on some version of a volume-of-fluid method. For example, there are codes that can model a dozen or more different materials in which each material is tracked by keeping a record of the volume fraction in each cell that is occupied by that material. There are competing interface tracking methods, most notably the level set method, but to the best of Professor Puckett's knowledge there are currently no DOE production codes that model the movement of a large number of distinct materials that are based on some version of the level set method, or any interface tracking method other than the volume-of-fluid method.

In all of Puckett's work with Pilliod in [26] they demonstrated that the algorithms were secondorder accurate by making multiple computations of a given problem on successively refined grids, each grid a factor of two finer than the grid which they had used in the previous computation. This allowed them to compute the convergence rate and hence the accuracy of the algorithm for that particular problem. However, except in certain limited instances, they did not offer a proof that any of our algorithms were second-order accurate.

\subsubsection{A Proof of Second-Order Accuracy in the Max Norm of a Class of VOF Interface Recon-} struction Algorithms.

In [27] Professor Puckett addressed the absence of a proof for the first of the two separate algorithms that comprise a VOF interface tracking algorithm. In this paper he proved if a piecewise linear VOF interface reconstruction algorithm satisfied certain conditions, then it must be secondorder accurate in the max norm. Of the three norms researchers commonly use to asses the accuracy of algorithms in this area, the max norm is the strongest. Convergence in the max norm immediately implies that the method also converges in the one and two norms. However, it almost always more difficult to prove convergence of a numerical method in the max norm. In fact, some methods fail to converge in the max norm but do converge in the one or two norm, or both.

Volume-of-fluid methods have been used by researchers to track material interfaces since at least the early 1970's. A variety of VOF algorithms have been developed for modeling everything from flame propagation to dendritic solidification (e.g., the Stefan problem). Volume-of-fluid methods were among the first interface tracking algorithms to be implemented in codes originally developed at the DOE National Laboratories and subsequently released to the general public that were capable of tracking material interfaces in a variety of complex material flow problems and they continue to be widely used at these institutions as well as by the general scientific community. Yet Professor Puckett is aware of only one other paper in this field that contains a proof of the accuracy of a VOF interface reconstruction method.

It is also important to recognize that computational science - and in particular the field of interface reconstruction and interface tracking - is filled with ad hoc methods for which there is no proof the method will converge for all problems that satisfy certain conditions. In [27] Professor Puckett precisely specified conditions under which a piecewise linear VOF interface reconstruction algorithm will converge at a second-order rate in the max norm. The most important of these conditions relates the cell size $h$ of the square grid that covers the (two-dimensional) computational domain to the maximum curvature $\kappa_{\max }$ of the interface:

$$
h \leq \min \left\{C_{h}\left(\kappa_{\max }\right)^{-1},\left(\kappa_{\max }\right)^{-2}\right\}
$$


where

$$
C_{h} \stackrel{\text { def }}{=} \frac{\sqrt{2}-1}{2 \sqrt{3}} .
$$

The condition in (1) is important because it relates a computational parameter, namely the cell size $h$, to a property of the interface, the curvature $\kappa$, which is invariant under rotations and translations of the coordinate system. This is particularly useful as a criterion in a computation with adaptive mesh refinement, since as the interface evolves with time the curvature in some regions of the computation may increase, requiring a finer grid in that region. Professor Puckett is not aware of any constraints similar to (1) in the (vast!) literature on interface tracking algorithms.

\subsubsection{A New Second-Order Accurate Volume-of-Fluid Interface Reconstruction Algorithms.}

In [27] Professor Puckett proved if a VOF interface reconstruction method satisfies certain conditions then it is second-order accurate in the max norm, but he did not produce or describe any such method. However the proof in [27] is constructive. Therefore, in a follow-on paper [28] Professor Puckett introduced a new piecewise linear VOF interface reconstruction method that is based on the criteria for second-order accuracy presented in [27] and demonstrated with a computational example that it is second-order accurate in the max norm. (Note however, since it satisfies the conditions set forth in [27], the proof in [27] guarantees this new VOF interface reconstruction algorithm will be second-order accurate in the max norm.) This new algorithm is quite different from other versions of piecewise linear VOF interface reconstruction method that purport to be second-order accurate.

\subsubsection{An Improved Constraint on h for Second-Order VOF Interface Reconstruction Algorithms.}

Note that the CFL-like constraint in (1) consists of two constraints:

$$
\begin{aligned}
& h \leq C_{h}\left(\kappa_{\max }\right)^{-1}, \\
& h \leq\left(\kappa_{\max }\right)^{-2},
\end{aligned}
$$

and that the second of these two constraints is considerably more restrictive as the curvature $\kappa$ of the interface increases: $\kappa \rightarrow \infty$. Professor Puckett submitted an account of this work to the journal "Communications in Applied Mathematics and Computational Science", in which he proves the more restrictive constraint in (3) is unnecessary. Thus, for a given interface, one can reconstruct the interface to second-order in $h$ using a larger cell size $h$ than dictated by (1). The original research article describing this work [29] has been accepted for publication in "Communications in Applied Mathematics and Computational Science" and should appear sometime in 2013.

\subsubsection{Shock Pairs.}

Professor Puckett has also submitted a paper [14] to the Springer journal "Shock Waves" with his coauthor and mentor in the area of shock waves refracting at a gas interface, Dr. L. F. Henderson from Sydney University (retired). In this paper they identify a new type of shock refraction, which they call a shock pair. In their work Henderson and Puckett present both experimental and computational evidence that there are both regular and irregular shock pairs and derive a formula for predicting regular shock pairs. Dr. Henderson and Professor Puckett have received and responded to one round of reviews from two reviewers, after which one of the reviewers recommended publishing the papers at that time. However, the second reviewer recommends publishing the paper only after several technical details are clarified. Dr. Henderson and Professor Puckett are currently in the process of addressing these technical issues and hope to have the paper accepted for publication sometime in 2013. 


\subsection{Work Performed by Sarah Williams.}

\subsubsection{Comparison of a Fast Multipole Method-Based Poisson Solver to the Multigrid Method.}

With Professor Puckett's guidance Sarah Williams, who at the time was a graduate student in the GGAM at UCD, implemented an algorithm for approximating solutions of the Poisson equation

$$
\Delta \phi=f
$$

that is based on the Fast Multipole Method (FMM) [13]. Williams' work is based on previous work by L. Greengard and his collaborators [10] and [12].

The Poisson equation (4) is ubiquitous in science and engineering and the numerical solution of the Poisson equation is often (almost always!) the most expensive and least robust part of numerical algorithms used in science and engineering applications. In particular, in a projection method for approximating solutions of the incompressible Euler or incompressible Navier-Stokes equations one must solve a version of (4) one or more times per time step. The original projection method is due to Chorin [8] and much of the development of modern, high-order versions of these methods is due to Drs. Phillip Colella and John Bell and their coworkers at LBNL (e.g., $[2,3,4,7]$ ).

Typically these researchers use some form of iterative Poisson solver (e.g., Multigrid (MG) or Preconditioned Conjugate Gradient) in their projection methods. However, for some problems iterative methods can take an unreasonably large number of iterations to converge, or even fail to converge. The goal of Sarah's work was to develop a direct rather than iterative solver that is guaranteed to produce an accurate approximation to the solution of (4) in a fixed amount of time that can can be determined a priori, even when iterative methods fail. In Sarah's Master's thesis [38] she describes her implementation of the FMM and compared the efficiency of this algorithm to the efficiency of MG on a specific class of test problems.

2.2.2. Development of a Fast Multipole Method-Based Poisson Solver with Applications to Approximating Solutions of the Incompressible Euler and Navier-Stokes Equations.

At Professor Puckett's suggestion Sarah Williams spent the summer of 2005 working with Dr. Ann Almgren in CCSE implementing her version of the (two dimensional) FMM in the FORTRAN and $\mathrm{C}++$ software infrastructure in which CCSE researchers have implemented their projection methods. Williams then used the resulting method to examine its performance on problems for which the MG solver CCSE researchers typically use in their projection method either fails or uses an unusually large number of iterations. This work is reported on in LBNL Technical Report [40].

\subsubsection{Numerical Methods for the Stochastic Landau-Lifshitz Navier-Stokes Equations.}

By the end of her stay at LBNL working with Dr. Almgren and interacting with Dr. Almgren's coworkers in CCSE Sarah decided she wanted to change the direction of her PhD thesis research. She liked the research environment at LBNL and elected to stay there and work on a topic suggested by Dr. John Bell, namely the development of numerical methods for the stochastic Navier-Stokes Equations. With the guidance of Dr. Bell and his collaborator Professor Alejandro L. Garcia from San Jose State University Sarah wrote a PhD thesis entitled "A Multiscale Hybrid Algorithm for Fluctuating Hydrodynamics", which was filed in June 2007 [39]. During the first year of her work on her PhD thesis (i.e.; Academic Year 2005-2006) Sarah was partially supported with funds from DE-FG02-03ER25579, with the remainder of her support coming from Professor Puckett's SciDAC grant DE-FC02-01ER25473. During her second and final year at LBNL, she was supported with a Dissertation Year Fellowship from the University of California, Davis and a \$10,000 scholarship known as a PEO (Philanthropic Educational Organization) Scholarship. Sarah is currently an Instructor in the Department of Mathematics at Duke University. The following publications have resulted from her work with Drs. Bell and Garcia: [6] and [37]. 


\subsection{Analysis of the Temporal Accuracy of Interface Tracking Algorithms that are} Second-Order Accurate in Space.

In December 2005, under the guidance of Professor Puckett, Chris Algieri, who at the time was a graduate student in the GGAM at UCD, completed his MS thesis [1]. Algieri's work focused on the numerical solution of the Stefan problem, which is the heat equation with an interface that is held at a fixed temperature, say $T=0$, at which the material being modeled solidifies. In his thesis Algieri examined the accuracy in time of two different algorithms, each of which have a stencil for the spatial derivatives in a neighborhood of the material interface that is designed to yield a second-order accurate solution to the associated Poisson problem; i.e., the problem for which the time derivative vanishes. (This can be thought of as the corresponding steady state problem.)

This is currently an active area of research for several reasons. One is that some algorithms that produce second-order accurate results for the associated Poisson problem are only first-order accurate in time, even if one uses a second-order accurate time stepping algorithm such as CrankNicolson. Furthermore, even those algorithms that are second-order accurate in time, tend to be unstable if one uses a standard second-order accurate time stepping algorithms such as CrankNicolson. This problem was first noticed by Johansen and Colella in [15] and their solution to this problem involved using a more stable time stepping procedure due to Twizell, Gumel and Arigu [36]. Our ANAG colleagues at LBNL have since published several additional papers in this area: [9], [18] and [30]. In his MS thesis [1] Algieri studied algorithms for numerically analyzing the stability of operators that are based on second-order accurate spacial stencils and determined criteria for deciding if a given method will be second-order accurate in time.

\section{Work Performed by Professor Miller and Members of His Research Group}

\subsection{The Determination of the Symmetry Invariants of the Hyperelastic Stress (Strain) Function.}

In [19] Professor Miller determined the symmetry invariants of the hyperelastic stress (strain) function using modern approaches to computational invariant theory. In this work Professor Miller identified for the first time a minimal basis for all invariant stress (strain) functions.

\subsection{The Determination of the Symmetry Invariants of the Hyperelastic Stress (Strain) Function.}

In resistive magnetohydrodynamics (MHD) there is an elliptic problem that is not stable for any straightforward discretization of the problem. In [11] Professor Miller and several ANAG collaborators developed a regularization technique, which stabilized the solver, and for which they were able to demonstrate second-order accurate convergence in regular domains.

\subsection{Development of New Solvers for the Stochastic Langevin Equations and New Fluid-Particle Coupling Methods.}

In collaboration with Dr. Trebotich and other ANAG researchers Professor Miller developed hybrid particle-continuum methods for bead-rod and bead-spring abstractions of polymer solutions. This work involved the development of new solvers for the stochastic Langevin equations and new fluid-particle coupling methods. It is reported on in the following publications: [16], [17], [33], [34] and [35].

\subsection{The Development of Efficient Solvers for the Oldroyd-B Equations of Continuum Viscoelasticity.}

In collaboration with his graduate student Andrew Nonaka in the Department of Applied Science at UCD, together with Dr. David Trebotich and other ANAG researchers at LBNL, Professor Miller developed efficient solvers for the Oldroyd-B equations of continuum viscoelasticity. This approach 
recovers the correct solution in both viscous and elastic limits. Furthermore, in these solvers the time step is governed by an effective wave speed that for some problems is close to the advective speed, which may be many orders of magnitude less than the true elastic wave speed. This work is reported on in the following publications: [23], [32] and [31].

The work in Dr. Nonaka's PhD thesis [22], which he completed in 2007, is closely related to the work just described. Dr. Nonaka's thesis research was partially supported by DOE grant \# DE-FG02-03ER25579. Dr. Nonaka is now a Research Scientist in CCSE at LBNL.

\subsection{Other Graduate Students Supported by Professor Miller.}

In addition to Dr. Nonaka, Professor Miller also supported two other graduate students, Michael McConnehey and Thomas Marshall, in the Department of Applied Science at UCD while they worked on their Master's degrees under his direction.

\section{Collaborative Research Between Professors Miller and Puckett}

Professors Miller and Puckett have developed a new and very efficient method for domain coupling that scales efficiently and is several orders of magnitude faster in computing time than a recently published algorithm for performing the same computation. This is an outgrowth of Professor Miller's research, reported on in [20], in which he developed iterative boundary potential algorithms for a special class of Poisson problems. The research by Professor Miller described in [20] was also supported in part by DOE grant \# DE-FG02-03ER25579.

In December 2011 Professors Miller and Puckett submitted an original research article for publication in the Journal of Computational Physics describing this work.

This article was published on February 15, 2013 in the Journal of Computational Physics [21]. 


\section{List of Technical Reports, Conference Proceedings, Journal Articles and Theses}

Work reported in the following DOE Laboratory Technical Reports, Conference Proceedings, Journal Articles and MS \& PhD Theses were supported in part by DOE Grant DE-FG02-03ER25579. (Articles currently under review by a peer-reviewed journal are not included.)

DOE-UCDAVIS-25579-00: Final Report for DOE Grant DE-FG02-03ER25579: "Development of High-Order Accurate Interface Tracking Algorithms and Improved Constitutive Models for Problems in Continuum Mechanics with Applications to Jetting"

DOE-UCDAVIS-25579-01: C. A. Algieri. A Comparison of Two Methods to Solve the 1-D Heat Equation on an Irregular Domain in the Context of the Stefan Problem. MS Thesis, Graduate Group in Applied Mathematics University of California, Davis, December 2005.

DOE-UCDAVIS-25579-02: D. T. Graves, D. P. Trebotich, G. H. Miller, and P. Colella. An Efficient Solver for the Equations of Resistive MHD with Spatially-Varying Resistivity. J. Comput. Phys., 227(10):4797-4804, May 2008.

DOE-UCDAVIS-25579-03: B. Kallemov, G. H. Miller, and D. P. Trebotich. A HigherOrder Approach to Fluid-Particle Coupling in Microscale Polymer Flows. In Nanotechnology 2008: Microsystems, Photonics, Sensors, Fluidics, Modeling, and Simulation, v 3, Boston, MA, June 1-5, 2008, pages 425-428, 2008. (Also published as LLNL Technical Report LLNL-PROC-402330.).

DOE-UCDAVIS-25579-04: B. Kallemov, G. H. Miller, and D. P. Trebotich. A Duhamel Approach for the Langevin Equations with Holonomic Constraints. Mol. Simulat., 35(6):440447, 2009.

DOE-UCDAVIS-25579-05: G. H. Miller. Minimal Rotationally-Invariant Bases for Hyperelasticity. SIAM J. Appl Math., 64(6):2050-2075, August 2004. (Also published as LBNL Technical Report LBNL-55531.)

DOE-UCDAVIS-25579-06: G. H. Miller. An iterative boundary potential method for the infinite domain Poisson problem with interior Dirichlet boundaries. J. Comput. Phys., 227(16):7417-7928, August 2008.

DOE-UCDAVIS-25579-07: A. J. T. Nonaka. A Higher-Order Upwind Method for Viscoelastic Flow. PhD Thesis, Department of Applied Science, University of California, Davis, 2007.

DOE-UCDAVIS-25579-08: A. J. T. Nonaka, D. P. Trebotich, G. H. Miller, D. T. Graves, and P. Colella. A higher-order upwind method for viscoelastic flow. Comm. App. Math. Comput. Sci., 4:57-83, 2009.

DOE-UCDAVIS-25579-09: J. E. Pilliod and E. G. Puckett. Second-Order Accurate VolumeOf-Fluid Algorithms For Tracking Material Interfaces. J. Comput. Phys., 199(2):465-502, September 2004.

DOE-UCDAVIS-25579-10: E. G. Puckett. On the Second-Order Accuracy of Volumeof-Fluid Interface Reconstruction Algorithms: Convergence in the Max Norm. CAMCoS, $5(1): 99-148,2010$. 
DOE-UCDAVIS-25579-11: E. G. Puckett. A Volume-of-Fluid Interface Reconstruction Algorithm that is Second-Order Accurate in the Max Norm. CAMCoS, 5(2):199-220, 2010.

DOE-UCDAVIS-25579-12: E. G. Puckett. On the Second-Order Accuracy of Volumeof-Fluid Interface Reconstruction Algorithms II An Improved Constraint on the Cell Size $C A M C o S$, (Accepted).

DOE-UCDAVIS-25579-13: D. Trebotich, P. Colella, and G. H. Miller. A stable and convergent scheme for viscoelastic flow in contraction channels. J. Comput. Phys., 205(1):315342, May 2005.

DOE-UCDAVIS-25579-14: D. P. Trebotich, P. Colella, G. H. Miller, A. J. T. Nonaka, T. H. Marshall, S. Gulati, and D. Liepmann. A Numerical Algorithm for Complex Biological Flow in Irregular Microdevice Geometries. In Technical Proceedings of the 2004 Nanotechnology Conference and Trade Show, March 7-11, 2004, Boston, MA, volume 2, pages 470-473. Nano Science and Technology Institute, Cambridge, MA, CRC Press, June 2007. (Also published as LLNL Technical Report UCRL-CONF-201479.).

DOE-UCDAVIS-25579-15: D. P. Trebotich, G. H. Miller, P. Colella, D. T. Graves, D. F. Martin, and P. O. Schwartz. A Tightly Coupled Particle-Fluid Model for Dna-Laden Flows in Complex Microscale Geometries. In Computational Fluid and Solid Mechanics 2005, pages 1018-1022, 2005. (Also published as LLNL Technical Report UCRL-CONF-208132.).

DOE-UCDAVIS-25579-16: D. P. Trebotich and G. H. Miller. Modeling and simulation of DNA flow in a microfluidic-based pathogen detection system. In Proceedings of the $3^{\text {rd }}$ Annual International IEEE/EMBS Special Topic Conference on Microtechnologies in Medicine and Biology, pages 353-355, Kahuku, Oahu, Hawaii ú, May 12-15 2005.

DOE-UCDAVIS-25579-17: D. P. Trebotich, G. H. Miller, and M. D. Bybee. A Penalty Method to Model Particle Interactions in DNA-Laden Flows. J Nanosci. Nanotechno., 8(7):3749-3756, July 2008.

DOE-UCDAVIS-25579-18: S. A. Williams. A Comparison of a Fast Multipole MethodBased Poisson Solver to a Multigrid Method. MS Thesis, Graduate Group in Applied Mathematics, University of California, Davis, December 2004.

DOE-UCDAVIS-25579-19: S. A. Williams. A Multiscale Hybrid Algorithm for Fluctuating Hydrodynamics. PhD Thesis, Graduate Group in Applied Mathematics, University of California, Davis, June 2007.

DOE-UCDAVIS-25579-20: S. A. Williams, A. S. Almgren, and E. G. Puckett. On Using a Fast Multipole Method-based Poisson Solver in an Approximate Projection Method. Technical Report LBNL-59934, Lawrence Berkeley National Laboratory, March 2006. 


\section{REFERENCES}

[1] C. A. Algieri. A Comparison of Two Methods to Solve the 1-D Heat Equation on an Irregular Domain in the Context of the Stefan Problem. MS Thesis, Graduate Group in Applied Mathematics, University of California, Davis, December 2005.

[2] A. S. Almgren, J. B. Bell, P. Colella, L. H. Howell, and M. L. Welcome. A Conservative Adaptive Projection Method for the Variable Density Incompressible NavierStokes Equations. SIAM J. Sci. Comput., 142(1):1-46, May 1998.

[3] A. S. Almgren, J. B. Bell, P. Colella, and T. Marthaler. A Cartesian Grid Projection Method for the Incompressible Euler Equations in Complex Geometries. SIAM J. Sci. Comput., 18(5):1289-1309, May 1997.

[4] J. B. Bell, P. Colella, and H. M. Glaz. A Second-Order Projection Method for the Incompressible Navier-Stokes Equations. J. Comput. Phys., 85(2):257-283, December 1989.

[5] J. B. Bell, C. N. Dawson, and G. R. Shubin. An Unsplit, Higher Order Godunov Method for Scalar Conservation Laws in Multiple Dimensions. J. Comput. Phys., 74(1):1-24, January 1988.

[6] J. B. Bell, A. L. Garcia, and S. A. Williams. Numerical methods for the stochastic Landau-Lifshitz Navier-Stokes equations. Phys. Rev. E, 76(016708):1-12, August 2007.

[7] J. B. Bell and D. L. Marcus. A Second-Order Projection Method for Variable Density Flows. J. Comput. Phys., 101(2):334-348, August 1992.

[8] A. J. Chorin. Numerical solution of the Navier-Stokes equations. Math. Comput., 22(104):745-762, 1968.

[9] P. Colella, D. Graves, and J. A. Greenough. A Second-Order Method for Interface Reconstruction in Orthogonal Coordinate Systems. Technical Report LBNL-45244, Lawrence Berkeley National Laboratory, January 2002.

[10] F. Ethridge and L. Greengard. A New Fast-Multipole Accelerated Poisson Solver in Two Dimensions. SIAM J. Sci. Comput., 23(3):741-760, 2001.

[11] D. T. Graves, D. P. Trebotich, G. H. Miller, and P. Colella. An Efficient Solver for the Equations of Resistive MHD with Spatially-Varying Resistivity. J. Comput. Phys., 227(10):4797-4804, May 2008.

[12] L. F. Greengard and J.-Y. Lee. A Direct Adaptive Poisson Solver of Arbitrary Order Accuracy. J. Comput. Phys., 125(2):415-424, May 1996.

[13] L. F. Greengard and V. Rockhlin. A Fast Algorithm for Particle Simulations. J. Comput. Phys., 73(2):325-348, December 1987.

[14] L. F. Henderson and E. G. Puckett. The Refraction of Shock Pairs. Shock Waves, 2013. (submitted).

[15] H. S. Johansen and P. Colella. A Cartesian Grid Embedded Boundary Method for Poisson's Equation on Irregular Domains. J. Comp. Phys., 147(1):60-85, November 1998.

[16] B. Kallemov, G. H. Miller, and D. P. Trebotich. A Higher-Order Approach to Fluid-Particle Coupling in Microscale Polymer Flows. In Nanotechnology 2008: Microsystems, Photonics, Sensors, Fluidics, Modeling, and Simulation - Technical Proceedings of the 2008 NSTI Nanotechnology Conference and Trade Show, volume 3 of Nanotech Conference Technical Proceedings, pages 425-428, Boston, MA, June 1-5, 2008 2008. NanoScience and Technology Inst. (Also published as LLNL Technical Report LLNL-PROC-402330.).

[17] B. Kallemov, G. H. Miller, and D. P. Trebotich. A Duhamel Approach for the Langevin Equations with Holonomic Constraints. Mol. Simulat., 35(6):440-447, 2009.

[18] P. McCorquodale, P. Colella, and H. S. Johansen. A Cartesian Grid Embedded Boundary Method for the Heat Equation on Irregular Domains. J. Comput. Phys., 173(2):620-635, November 2001.

[19] G. H. Miller. Minimal Rotationally-Invariant Bases for Hyperelasticity. SIAM J. Appl Math., 64(6):2050-2075, August 2004. (Also published as LBNL Technical Report LBNL-55531.).

[20] G. H. Miller. An iterative boundary potential method for the infinite domain Poisson problem with interior Dirichlet boundaries. J. Comput. Phys., 227(16):7417-7928, August 2008.

[21] G. H. Miller and E. G. Puckett. A Neumann-Neumann preconditioned iterative substructuring approach for computing pressure in multifluid problems with surface tension. J. Comput. Phys., 235(0):683-700, 15 February 2013.

[22] A. J. T. Nonaka. A Higher-Order Upwind Method for Viscoelastic Flow. PhD Thesis, Department of Applied Science, University of California, Davis, 2007.

[23] A. J. T. Nonaka, D. P. Trebotich, G. H. Miller, D. T. Graves, and P. Colella. A higher-order upwind method for viscoelastic flow. Comm. App. Math. Comput. Sci., 4:57-83, 2009.

[24] J. E. Pilliod. An Analysis of Piecewise Linear Interface Reconstruction Algorithms for Volume-of-Fluid Methods. MS Thesis, Graduate Group in Applied Mathematics, University of California, Davis, September 1992.

[25] J. E. Pilliod. A Second-Order Unsplit Method for Modeling Flames in Two-Dimensional Compressible Flow. PhD Thesis, Graduate Group in Applied Mathematics, University of California, Davis, September 1996.

[26] J. E. Pilliod and E. G. Puckett. Second-Order Accurate Volume-of-Fluid Algorithms for Tracking Material Interfaces. J. Comput. Phys., 199(2):465-502, September 2004. 
[27] E. G. Puckett. On the Second-Order Accuracy of Volume-of-Fluid Interface Reconstruction Algorithms: Convergence in the Max Norm. CAMCoS, 5(1):99-148, 2010.

[28] E. G. Puckett. A Volume-of-Fluid Interface Reconstruction Algorithm that is Second-Order Accurate in the Max Norm. CAMCoS, 5(2):199-220, 2010.

[29] E. G. Puckett. On the Second-Order Accuracy of Volume-of-Fluid Interface Reconstruction Algorithms II: An Improved Constraint on the Cell Size. CAMCoS, 2013. Accepted for publication.

[30] P. O. Schwartz, M. Barad, P. Colella, and T. J. Ligocki. A Cartesian Grid Embedded Boundary Method for the Heat Equation and Poisson's Equation in Three Dimensions. J. Comput. Phys., 211(2):531-550, January 2006.

[31] D. Trebotich, P. Colella, and G. H. Miller. A stable and convergent scheme for viscoelastic flow in contraction channels. J. Comput. Phys., 205(1):315-342, May 2005.

[32] D. P. Trebotich, P. Colella, G. H. Miller, A. J. T. Nonaka, T. H. Marshall, S. Gulati, and D. Liepmann. A Numerical Algorithm for Complex Biological Flow in Irregular Microdevice Geometries. In Technical Proceedings of the 2004 Nanotechnology Conference and Trade Show, March 7-11, 2004, Boston, MA, volume 2, pages 470473. Nano Science and Technology Institute, Cambridge, MA, CRC Press, June 2007. (Also published as LLNL Technical Report UCRL-CONF-201479.).

[33] D. P. Trebotich, G. H. Miller, P. Colella, D. T. Graves, D. F. Martin, and P. O. Schwartz. A Tightly Coupled Particle-Fluid Model for Dna-Laden Flows in Complex Microscale Geometries. In Computational Fluid and Solid Mechanics 2005, pages 1018-1022, 2005. (Also published as LLNL Technical Report UCRL-CONF-208132.).

[34] D. P. Trebotich and G. H. Miller. Modeling and simulation of DNA flow in a microfluidic-based pathogen detection system. In Proceedings of the $3^{\text {rd }}$ Annual International IEEE/EMBS Special Topic Conference on Microtechnologies in Medicine and Biology, pages 353-355, Kahuku, Oahu, Hawaii ú, May 12-15 2005.

[35] D. P. Trebotich, G. H. Miller, and M. D. Bybee. A Penalty Method to Model Particle Interactions in DNA-Laden Flows. J Nanosci. Nanotechno., 8(7):3749-3756, July 2008.

[36] E. H. Twizell, A. B. Gumel, and M. A. Arigu. Second-Order, L0-Stable Methods for the Heat Equation with Time-Dependent Boundary Conditions. Adv. Comput. Math., 6(1):333-352, August 1996.

[37] S. A. Williams, J. B. Bell, and A. L. Garcia. Algorithm refinement for fluctuating hydrodynamics. Multiscale Model Sim, 6(4):1256-1280, February 2008.

[38] S. A. Williams. A Comparison of a Fast Multipole Method-Based Poisson Solver to a Multigrid Method. MS Thesis, Graduate Group in Applied Mathematics, University of California, Davis, December 2004.

[39] S. A. Williams. A Multiscale Hybrid Algorithm for Fluctuating Hydrodynamics. PhD Thesis, Graduate Group in Applied Mathematics, University of California, Davis, June 2007.

[40] S. A. Williams, A. S. Almgren, and E. G. Puckett. On Using a Fast Multipole Method-based Poisson Solver in an Approximate Projection Method. Technical Report LBNL-59934, Lawrence Berkeley National Laboratory, March 2006. 